\title{
Synchronous Gastric Adenocarcinoma and Perigastric Lymph Node Metastatic Squamous Cell Carcinoma with Unknown Primary: A Case Report
}

\author{
Bernard K Seshie, M.D. ${ }^{1}$, Ki Hyun Kim, M.D. ${ }^{3}$, Hyun Jung Lee, M.D. ${ }^{2}$, Si Hak Lee, M.D., Ph.D. ${ }^{3}$, Sun-Hwi Hwang, M.D., Ph.D. ${ }^{3}$ \\ ${ }^{1}$ Department of Surgery, Tema General Hospital, Tema, Ghana, ${ }^{2}$ Department of Pathology, Pusan National University Yangsan Hospital, ${ }^{3}$ Department of \\ Surgery and Research Institute for Convergence of Biomedical Science and Technology, Pusan National University Yangsan Hospital, Yangsan, Korea
}

We report a case of 65 years old man who was found on routine esophagogastroduodenoscopy to have gastric tumour. Histology revealed adenocarcinoma. Staging investigation with abdominal computed tomography (CT) confirmed gastric cancer of the antrum with lymph node enlargement at hepatoduodenal ligament. The patient underwent laparoscopic assisted distal gastrectomy with D2 lymphadenectomy. Pathology revealed intestinal type moderately differentiated tubular adenocarcinoma invading lamina propria (pTla). Four positive lymph nodes out of 34 did not show metastatic adenocarcinoma but rather Squamous Cell Carcinoma (SCC) and were positive for p63 and CK5/6 on immunohistochemistary. Primary site of SCC was not found. He received adjuvant chemotherapy with TS-1 $60 \mathrm{mg}$. After two years of follow-up he is asymptomatic and repeated EGD and abdominal CT Scan were normal. Herein, the authors report the case of early gastric cancer with synchronous metastatic SCC with unknown primary site.

Keywords: Adenocarcinoma, Metastasis, Perigastric lymph node, Synchronous, Squamous cell carcinoma
Received May 29, 2019

Revised July 29, 2019

Accepted August 29, 2019

Corresponding author

Sun-Hwi Hwang

Department of Surgery and Research Institute for Convergence of Biomedical Science and Technology, Pusan

National University Yangsan Hospital, 20 Geumo-ro, Mulgeum-up, Yangsan 50612, Korea

Tel: $+82-55-360-2124$

Fax: +82-55-360-2154

E-mail: shhwang@pnuyh.co.kr ORCID:

https://orcid.org/0000-0001-8058-0524

Bernard K Seshie and Ki Hyun Kim shared first authorship.

Copyright (c) 2020 The Journal of Minimally Invasive Surgery. All rights reserved.

This is an Open Access article distributed under the terms of the Creative Commons Attribution Non-Commercial License (http:/ creativecommons.org/licenses/by-nc/4.0/) which permits unrestricted non-commercial use, distribution, and reproduction in any medium, provided the original work is properly cited.

\section{INTRODUCTION}

Gastric cancer (GC) includes all malignant epithelial tumours of the stomach. Adenocarcinoma with various histological types (papillary, tubular, mucinous and poorly cohesive) account for $95 \%$ of cases. ${ }^{1}$ However, several unusual presentations have been reported in the literature review of this condition. These are in the form of unusual histological subtypes, configuration, spread or complication. ${ }^{2}$ Lymph nodes (LN) are the commonest site of metastasis in gastric cancer, and is a recognized prognostic factor and predictor of the disease recurrence. ${ }^{3}$

Perigastric LN are involved very early. However, cancers of the oesphagus, lung and the neck region can also metastasize to the perigastric nodes. ${ }^{4}$ Furthermore, metastic cancer in perigastric LN with unknown primary has been reported in the literature. ${ }^{5}$

Cancer of an unknown primary site (CUP) defined as a 
metastatic disease without an identifiable primary tumour after a full diagnostic work-up, represents 3 5\% of all invasive cancers, and the squamous cell carcinoma accounts for $15 \%$ of these cases. ${ }^{6}$ We report a case of an early antral gastric adenocarcinoma (pTla), with synchronous Squamous Cell Carcinoma (SCC) metastasis, in 4 out of 34 perigastric LN with occult primary SCC.

This study was approved by the Institutional Review Board of Pusan National University Yangsan Hospital. Informed consent to publish was obtained from the patient.

\section{CASE REPORT}

A 65 years old Korean male with no significant past medical and social history had a routine screening Esophagogastroduodenoscopy (EGD) at the Pusan National University Yangsan Hospital (Yangsan, South Korea), which revealed a broad-based mass with nodularity in the gastric antrum. Upon a review of the histology of the endoscopic biopsy from the antrum and the body, revealed a tubular moderately differentiated, intestinal type adenocarcinoma and a chronic gastritis respectively. A review of the staging abdominal computed to-

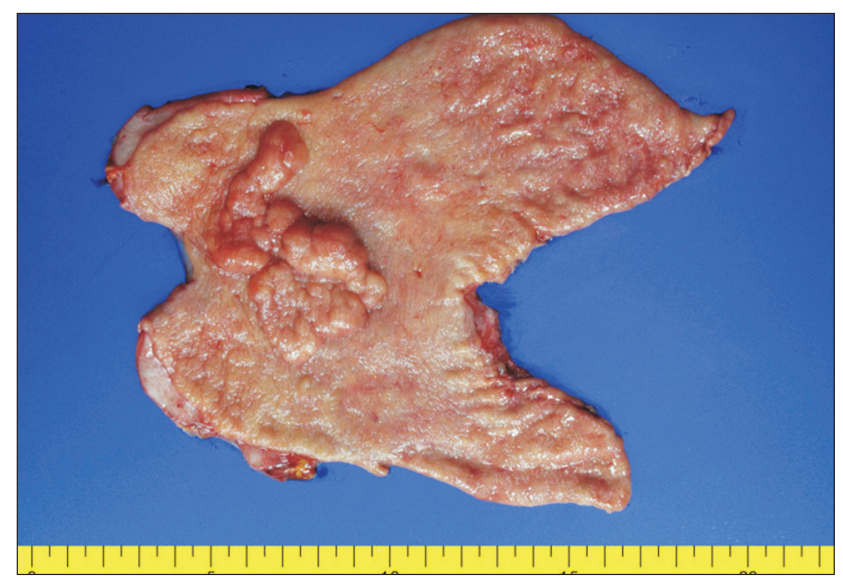

Fig. 1. Gross observation of the tumour. mography (CT) Scan demonstrated an ulcerofungating mass at the stomach antrum, with enlarged LN at the hepatoduodenal ligament and perigastric fat stranding. The positron emission tomography, fluorine-18 fluoro-2-2deoxy-D-glucose (PETCT: F-18 FDG) Torso (Skull base to thigh) were performed, and confirmed the GC of the antrum, with a lymph node enlargement at the hepatoduodenal ligament (cT4aN1M0).

The patient underwent a laparoscopic assisted distal gastrectomy with a D2 lymphadenectomy. The resected specimen measured $18.1 \mathrm{~cm}$ and $7.8 \mathrm{~cm}$ along the greater and lesser curvature respectively. Macroscopically, it exhibited relatively a well-defined fungating mass (Borrmann Type 1) measuring 7.2 $\times 4.1 \mathrm{~cm}$, and a well-defined yellow white flat lesion measuring $0.2 \times 0.2 \mathrm{~cm}$ at the antrum and the anterior wall of lower body respectively. The masses were $2.6 \mathrm{~cm}$ and $1.0 \mathrm{~cm}$ from the proximal and the distal resection margins respectively.

Histologically tumour was intestinal type, moderately differentiated tubular adenocarcinoma with areas of papillary well differentiated component (Fig. 1, 2). In this case the tumour invaded the lamina propria (pTla). Evidently, the proximal margin, distal margin and the anvil ring were free of the tumour with a safe margin of $2.6 \mathrm{~cm}$ proximal and distal of $1.0 \mathrm{~cm}$. No squamous differentiation was seen in the gastric cancer. There was no lymphatic, venous or perineural invasion at that time. In this sense, the immunohistochemistry study of the C-erbB2 was shown to be negative. A total of 34 LNs were retrieved from the specimen, 15 and 19 from the greater and lesser curvature respectively. None of the LNs contained metastatic deposits of the adenocarcinoma (pNO). However, 4 out of 34 ( 1 from greater curvature and 3 from the lesser curvature) retrieved LNs revealed the SCC, and were positive for p63 and CK5/6 on Immunohistochemistary (Fig. 2).

Chest CT Scan done a month after surgery showed mild pulmonary emphysema, left lung had two $2 \mathrm{~mm}$ sized sub pleural indeterminate nodule and LLL posterior basal segment, subsegmental atelectasis. After enough informed consent of various options for chemotherapy with hemato-oncologist, the patient treated to TS-1. He received 8 cycles of adjuvant che-

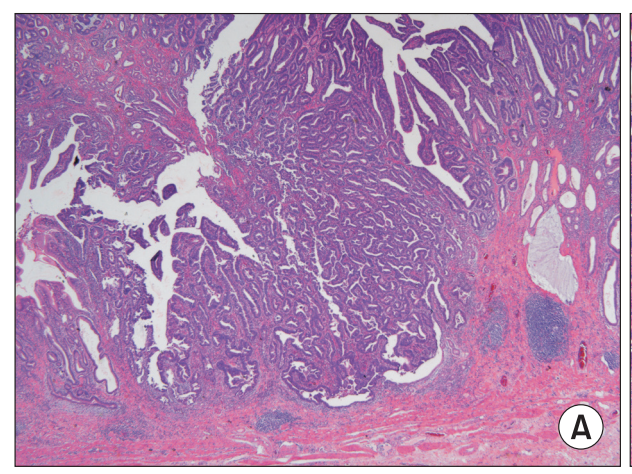

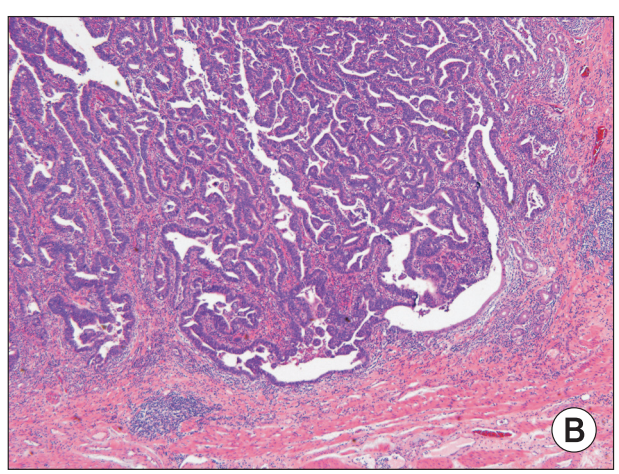

Fig. 2. Microscopic observation of the tumour (hematoxylin and eosin $\mathrm{A} \times 20$ and $B \times 40$ ) showing moderately differentiated tubular adenocarcinoma. 

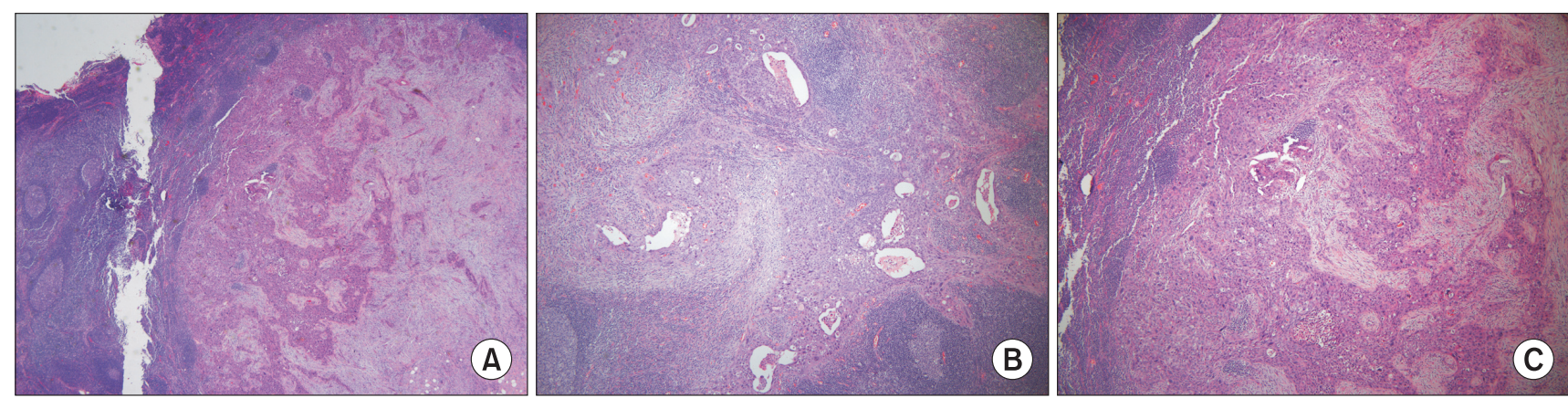

Fig. 3. Microscopic observation of the lymph nodes (hematoxylin and eosin $A \times 20, B$ and $C \times 40$ magnification) showing SCC with the keratin formation without any adenocarcinomatous component.

motherapy with oral TS-1 $60 \mathrm{mg}$. At last follow up two years after surgery, he is completely asymptomatic. The patient CT scan of the abdomen showed no evidence of the local tumour recurrence, and EGD revealed normal esophagus, subtotal gastrectomy state with no evidence of local tumour recurrence.

\section{DISCUSSION AND CONCLUSION}

The GC includes all the malignant epithelial tumours of the stomach. Adenocarcinoma with various histological types account for $95 \%$ of the cases. ${ }^{1}$ Several rare variants are recognized in the WHO classification, including an adenosquamous carcinoma and SCC. Unusual manifestations of gastric cancer have been reported. These are in the form of unusual histological subtypes, configuration, spread or complications related to this condition. ${ }^{2}$ Broadly speaking, the LNs are the commonest site of metastasis in gastric cancer, and is a recognized prognostic factor and predictor of disease recurrence. ${ }^{3}$ They correlate with the location and size of the tumour, depth of invasion, histological type and the Borrman type. The lymphatic drainage of the stomach is complicated and multidirectional, but the perigastric LNs are the first sites to be involved in early gastric cancers. However, cancers from other location such as esophagus, lungs and head and neck regions can also spread to the perigastric LNs. ${ }^{4}$

The present case is an early antral GC pTla, moderately differentiated tubular adernocarcinoma, Borrman Typel. The caes of a Tla tumour is defined as a tumour invading the lamina propria. Despite a tumour size of $7.3 \times 4.1 \mathrm{~cm}$, there was no metastasis deposit of the adenocarcinoma in the 34 retrieved regional LNs. Lymph node metastasis in an intramucosal early gastric cancer is variable but low. However, four out of the 34 lymph nodes had histological and immunohistochemistry features of SCC in this case.

SCC is a malignancy of epithelial origin capable of occurring in organs that are covered with a squamous epithelium.
Broadly speaking, the case of a non-melanoma skin cancer, head and neck cancer, esophageal cancer and non-small cell lung cancer account for the majority of these cases. ${ }^{8}$ The SCC as a variant of GC is an unusual histological subtype and rare in most cases. It is seen that this condition may occur as the primary SCC, adenosquamous carcinoma or as a collision tumour of the stomach. ${ }^{2}$ However, there was no histological evidence of either a squamous differentiation of the gastric antral tumour or a primary site of SCC in the resected specimen. In terms of the Synchronous SCC of the oesophagus and the adenocarcinoma of the stomach has also been documented in the literature, and the primary SCC of the esophagus has been shown to metastasize to perigastric LN. ${ }^{9}$ However, the preoperative and follow-up EGD in this patient did not revealed any pathology in the esophagus, and he is completely asymptomatic two years after surgery.

Furthermore, cancers of the lung and the neck region can also metastasize to the perigastric nodes. ${ }^{4}$ The prevailing discipline ntoes that the Chest and Abdominal CT Scan as well as the PET-CT: F-18 FDG Torso (Skull base to thigh) of our patient failed to demonstrate any lesion in the esophagus, lungs and head and neck region which were capable of metastazing to the perigastric lymph node in our patient.

We believe that adequate diagnostic work-up for the CUP has been carried out in this patient. Indeed, the FDG PET$\mathrm{CT}$ which was performed for this patient is considered to be very useful, and assisted the sensitive imaging modality for a patient with CUP. ${ }^{10}$ The Han et al. study revealed that, out of 120 patients with CUP, the FDG PET-CT was able to detect a primary tumour in 54 of the patients, representing $42.5 \%$ with a false positive rate of $7.5 \%$, and thus a primary tumour was found in only 5 cases of 60 unidentified cases during clinical follow-up between 2 to 45 months. ${ }^{10}$ This case presented a diagnostic dilemma, and will add to the existing literature as a case of synchronous gastric adenocarcinoma and metastatic SCC with unknown primary. 


\section{ORCID}

Bernard K Seshie, https://orcid.org/0000-0003-2540-790X

Ki Hyun Kim, https://orcid.org/0000-0002-8296-3307

Hyun Jung Lee, https://orcid.org/0000-0002-2995-6060

Si Hak Lee, https://orcid.org/0000-0002-1923-4777

Sun-Hwi Hwang, https://orcid.org/0000-0001-8058-0524

\section{AUTHORS' CONTRIBUTIONS}

Conceptualization: SHH. Formal analysis: BKS. Methodology: HJL, SHL. Writing-original draft: BKS, KHK. Writingreview and editing: KHK, SHH.

\section{CONFLICT OF INTEREST}

None.

\section{FUNDING}

None.

\section{ACKNOWLEDGMENTS}

None.

\section{REFERENCES}

1) Lochhead P, El-Omar E. Gastric Tumors: an overview. Atlas
Genet Cytogenet Oncol Haematol 2009;13:761-767.

2) Hwang SH, Lee JH, Kim K, et al. Primary squamous cell carcinoma of the stomach: A case report. Oncol Lett 2014;8:2122-2124.

3) Bedikian AY, Chen TT, Khankhanian N, et al. The natural history of gastric cancer and prognostic factors influencing survival. J Clin Oncol 1984;2:305-310.

4) Jeong GA, Kim HC, Kim HK, Cho GS. Perigastric lymph node metastasis from papillary thyroid carcinoma in a patient with early gastric cancer: the first case report. J Gastric Cancer 2014;14:215219.

5) Lee HS, Han HS, Lim SN, et al. Poorly differentiated neuroendocrine carcinoma in a perigastric lymph node from an unknown primary site. Cancer Res Treat 2012;44:271-274.

6) Pavlidis N, Pentheroudakis G. Cancer of unknown primary site: 20 questions to be answered. Ann Oncol 2010;21 Suppl 7:vii303307.

7) Hamilton SR, Aaltonen LA. World Health Organization classification of tumours : pathology and genetics of tumours of the digestive system. Lyon: IARC Publications; 2000.

8) Yan W, Wistuba, II, Emmert-Buck MR, Erickson HS. Squamous Cell Carcinoma - Similarities and Differences among Anatomical Sites. Am J Cancer Res 2011;1:275-300.

9) Li X, Lin S, Zhang Y, Wang H. Synchronous primary esophageal squamous cell carcinoma and gastric adenocarcinoma: analysis of 41 cases treated in a single institution. Sci Rep 2015;5:13335.

10) Han $A$, Xue J, Hu M, Zheng J, Wang $X$. Clinical value of $18 \mathrm{~F}-$ FDG PET-CT in detecting primary tumor for patients with carcinoma of unknown primary. Cancer Epidemiol 2012;36:470-475. 